\title{
Medial patello-femoral ligament (MPFL) reconstruction using suture anchors fixation: preliminary results
}

\author{
Filippo Calanna \\ Luca Pulici \\ Giulia Carimati \\ Alessandro Quaglia \\ Piero Volpi
}

Department of knee Orthopedic and Sports Traumatology Unit, Humanitas Research Hospital, Rozzano, Italy

Corresponding author:

Piero Volpi

Department of knee Orthopedic and Sports Traumatology Unit, Humanitas Research Hospital

Via Manzoni, 56

20089 Rozzano (MI), Italy

E-mail: piero.volpi@humanitas.it

\section{Summary}

Purpose: several surgical techniques have been described for the MPFL reconstruction, using many femoral and patellar fixation techniques and different grafts (autograft, allograft, synthetic).

The goal of our study is to evaluate the safety and efficacy of MPFL reconstruction using Juggerknot $^{\mathrm{TM}}$ (BIOMET, Warsaw, Indiana) suture anchors fixation with a clinical evaluation pre/post surgery of our patients with objective patellofemoral (PF) instability.

Methods: from January 2013 to March 2015, 19 patients underwent isolated MPFL reconstruction using Juggerknot ${ }^{\mathrm{TM}}$ (BIOMET, Warsaw, Indiana) suture anchors fixation. All patients were operated by the same surgeon using the same technique. Patients were evaluated by the same operator during pre surgery phase and at least at 6 months of follow up, using clinical evaluation (apprehension sign, patellar glide test, ROM) and 4 different scores (Tegner, Vas, Lysholm, Kujala). The possible complications and dislocation following surgery were investigated.

Results: no patellar dislocation and complications were found at follow up. A clinical evaluation improvement was recorded at follow up: all patients achieved a full range of knee motion; apprehension sign from $89 \%$ positivity (17 cases) decreased up to $11 \%$ ( 2 cases); glide test from $100 \%$ (19 cases) positivity decreased up to $0 \%$ ( 0 cases).

\begin{abstract}
Median VAS score decreased significantly $(p<0.05)$ from a median pre-operative value of 8 (min:5 max:10) to 2 (min:0 max:7). Mean Kujala score improved significantly $(p<0.05)$ from $65.23 \pm$ 18.64 pre-operatively to $94.69 \pm 6.40$. Mean Lysholm score improved significantly $(p<0.05)$ from $64.30 \pm 19.29$ pre-operatively to $94.72 \pm 4.02$. Mean Tegner score decreased from $6.15 \pm 1.06$ pre-operatively to $5.69 \pm 0.85$.

Conclusions: in this preliminary study, a clinical assessment of patients undergoing surgery with the Juggerknot ${ }^{\mathrm{TM}}$ (BIOMET, Warsaw, Indiana) suture anchor fixation in MPFL reconstruction, has shown promising results, revealing easy to apply, effective and safe for patients.
\end{abstract}

KEY WORDS: MPFL reconstruction, patellar instability, suture anchors, knee.

\section{Introduction}

The objective patellar dislocation (OPD) shows a yearly incidence of 6 cases over 100.000 , increasing $(29 / 100.000)$ in the age between 10 and 17 years ${ }^{1}$. It mainly affects young athletes of both sexes, with a light preponderance with females ${ }^{1}$. The conservative treatment is the first therapeutic approach in OPD, with a successful percentage of $37 \%^{2}$. According to OPD etiology (troclear dysplasia, patella alta, TT-TG> $20 \mathrm{~mm}$ ) different treatments can be used: trocleoplasty and anterior tibial tuberosity (ATT) transfer. In the last few years with medial patella-tibial ligament reconstruction (MPTL) ${ }^{3}$, the medial patella-femoral ligament (MPFL) reconstruction has proved to be an adequate procedure for OPD patients ${ }^{4}$. MPFL is the primary medial stabilizer of the patella ${ }^{5-8}$, limiting its lateral translation ${ }^{9}$. Several studies have shown that MPFL is always ruptured or deficient in case of acute dislocation or chronic patello-femoral (PF) instability $6,10,11$. The MPFL reconstruction can be performed either isolated or in association with trocleoplasty and ATT transfer. Several surgical techniques have been described for the MPFL reconstruction, using many femoral and patellar fixation techniques and different grafts (autograft, allograft, synthetic) ${ }^{12-15}$.

The goal of our study is to evaluate the safety and efficacy of MPFL reconstruction using Juggerknot ${ }^{\mathrm{TM}}$ (BIOMET, Warsaw, Indiana) suture anchors fixation with a clinical evaluation pre/post surgery of our patients. 


\section{Materials and methods}

From December 2012 to March 2015, 19 patients underwent isolated MPFL reconstruction using Jugger$\operatorname{knot}^{\mathrm{TM}}$ (BIOMET, Warsaw, Indiana) suture anchors fixation in our Knee surgery \& Sports Traumatology Department, Humanitas. All patients were operated by the same surgeon using the same technique. As required our research is ethically conduct according to international standards ${ }^{16}$.

Our inclusion criteria were: a) OPD patients that had not benefitted from the physiotherapy approach in the last 6 months; b) medial retinacular slackening due to frequent patellar dislocation; c) MPFL slackening with clinical and radiographic evidence; d) no association with other surgical techniques; e) normal troclea or troclear dysplasia (Type A, B); f) tuberculus sulcus angle between $0^{\circ}$ and $5^{\circ}+$, or TT-TG $<20 \mathrm{~mm}$.

Our exclusion criteria were: a) lack of neurologic or rheumatic pathologies; b) patella alta > 1.2 using Caton Deschamps Index or sagittal patella-femoral engagament (SPE) $<12.5 \%$.

Patients were evaluated by the same operator during pre surgery phase and at least at 6 months of follow up, using clinical evaluation (apprehension sign, patellar glide test, ROM) and 4 different scores (Tegner, Vas, Lysholm, Kujala). Number, causes and familiarity with the dislocation episodes were investigated and also the possible complications and dislocation following surgery.

\section{Surgical technique}

Our surgical technique is original, not similar to the others already published.

Once re-evaluated the patellar instability under anaesthesia, the semitendinosus muscle tendon shall be harvest (Figs. 1, 2). A parapatellar medial ap-

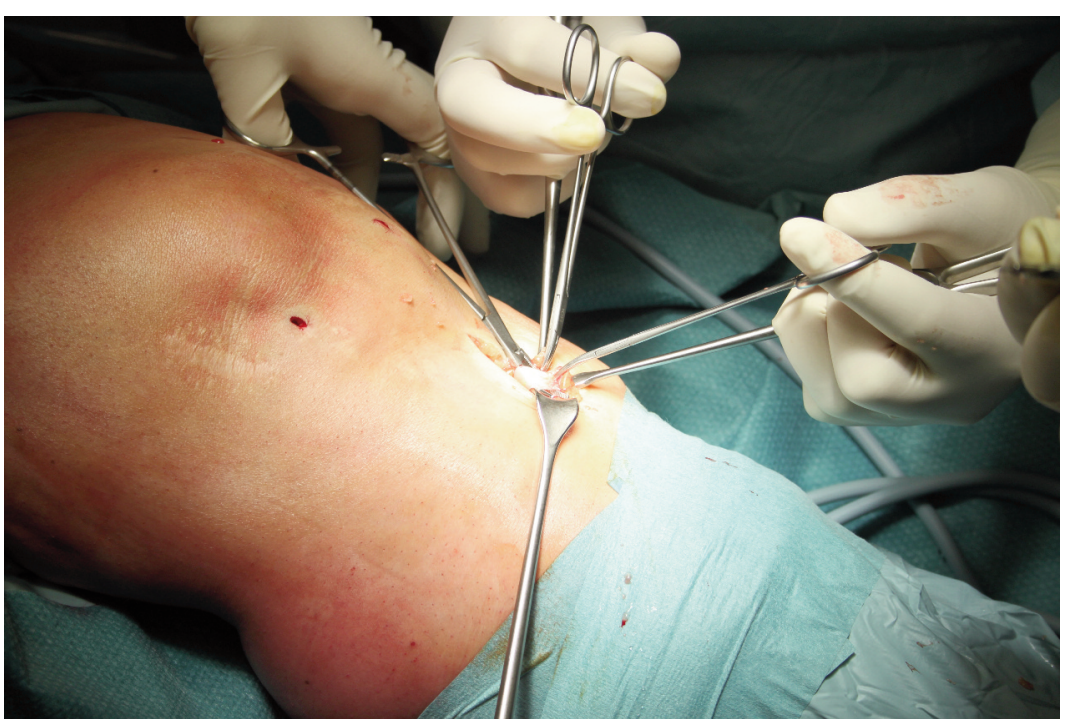

Figure 1. The harvest of the graft.

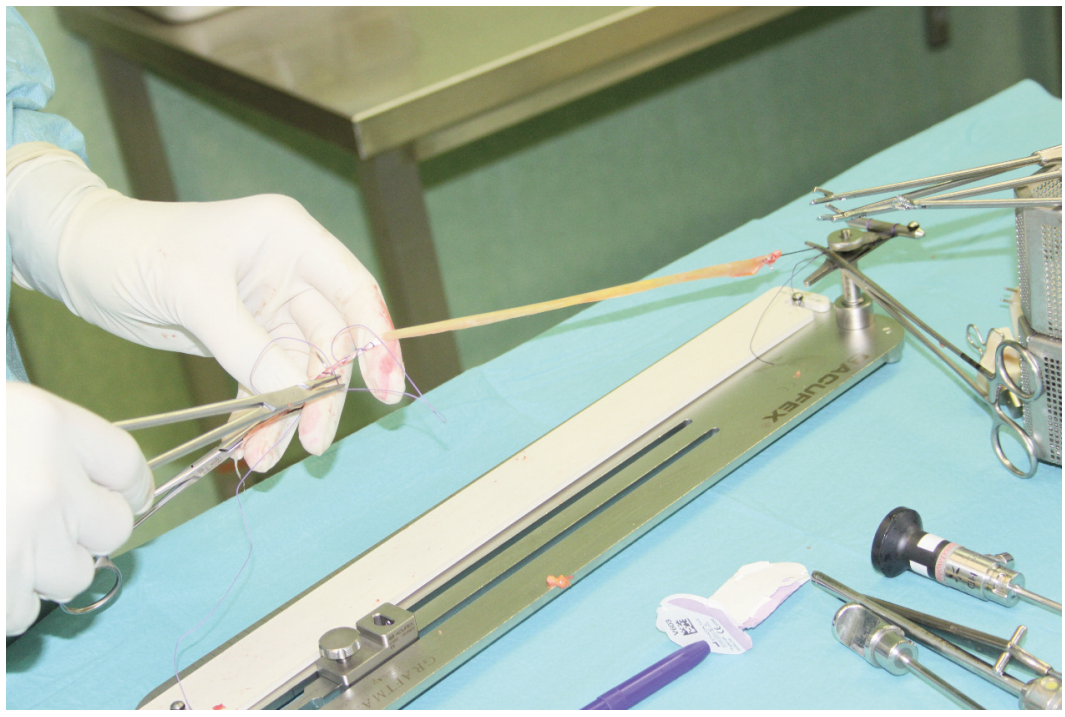

Figure 2. Neo medial patello femoral ligament (MPFL) preparation. 
proach is used and a sulcus on the medial border of the patella is done to fix the graft.

With special instruments drill a tunnel of $1.4 \mathrm{~mm}$ in diameter for the positioning of the three Juggerknot ${ }^{\mathrm{TM}}$ (BIOMET, Warsaw, Indiana) anchors at the medial patellar edge (Figs. 3, 4).

Under radiological control locate the correct positioning of the femoral tunnel at the level of the Schottle's point, where two guides in opposite directions are placed. Use a guide wire of about $5 \mathrm{~mm}$ proximal to the first Juggerknot ${ }^{\mathrm{TM}}$ (BIOMET, Warsaw, Indiana) anchor and approximately $5 \mathrm{~mm}$ distal to the second Juggerknot ${ }^{\mathrm{TM}}$ (BIOMET, Warsaw, Indiana) anchor (Figs. 5, 6).

The last surgical phase consists in anchoring the graft to the patellar and femoral insertion (Figs. 7, 8). After its patellar positioning, the surgeon fix the graft at the femoral level with the limb at $30^{\circ}$ of flexion.

\section{Post surgery rehabilitation}

Patients were dismissed two days after surgery with partial weight bearing using crutches. The brace was kept for 4 weeks gradually increasing flexibility to $60^{\circ}$ in the first two weeks and $90^{\circ}$ in the following two weeks. Vascular stocking and anti-thrombotic therapy were stopped after a month from surgery. The physiotherapy treatment in water in association with a use of stationary bicycle, quadriceps isometric strengthening exercises and biceps stretching were recommended from the second month after surgery. The schedule continues with an increasing muscle activity through isometric, isotonic, proprioceptive exercises together with functional evaluation tests. Return to sports is suggested after 5/6 months following surgery depending on the patient's amount of physical activity before operation.

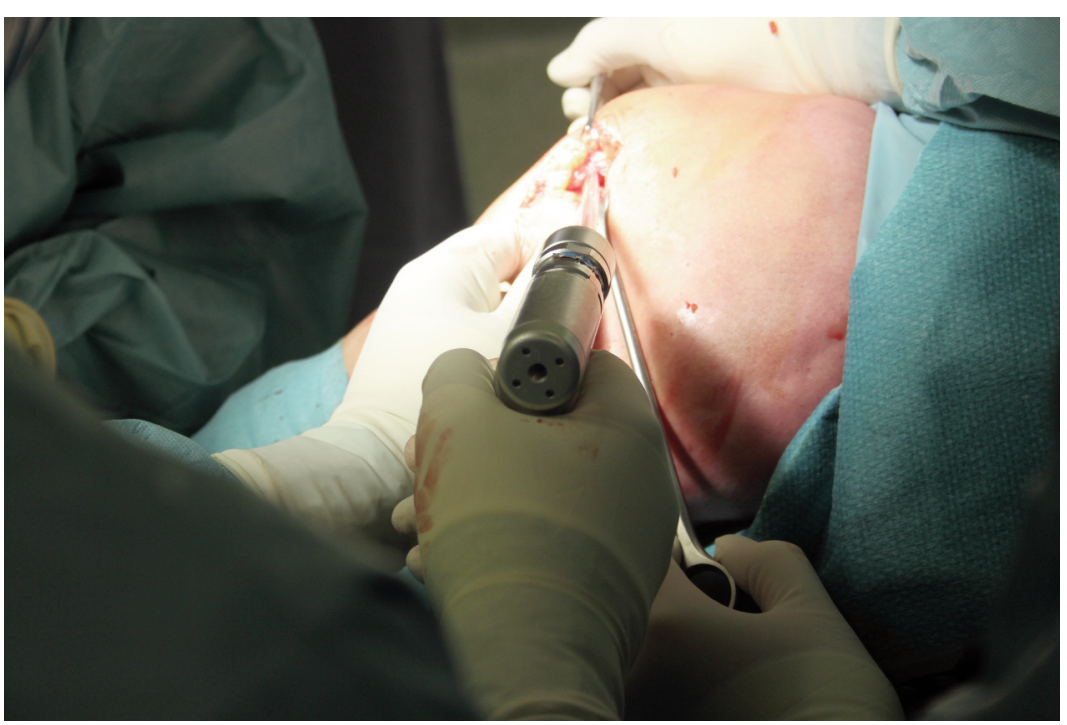

Figure 3. Drilling a tunnel of $1.4 \mathrm{~mm}$ in diameter for the positioning of the three anchors at the medial patellar edge.

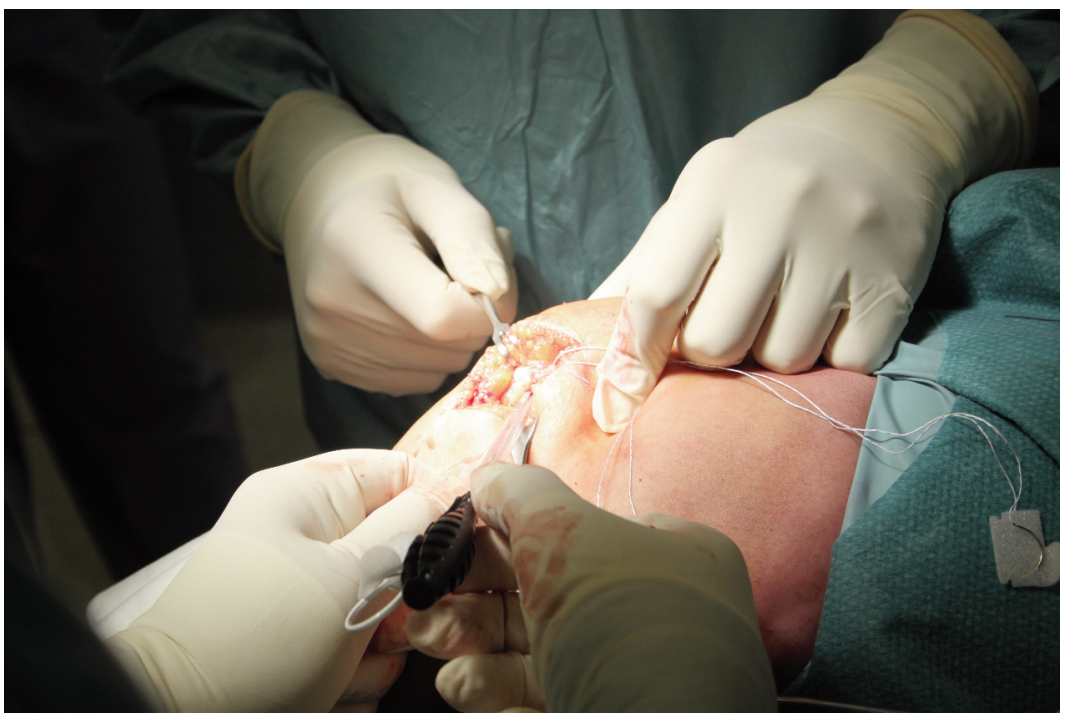

Figure 4. Anchors patellar positioning. 


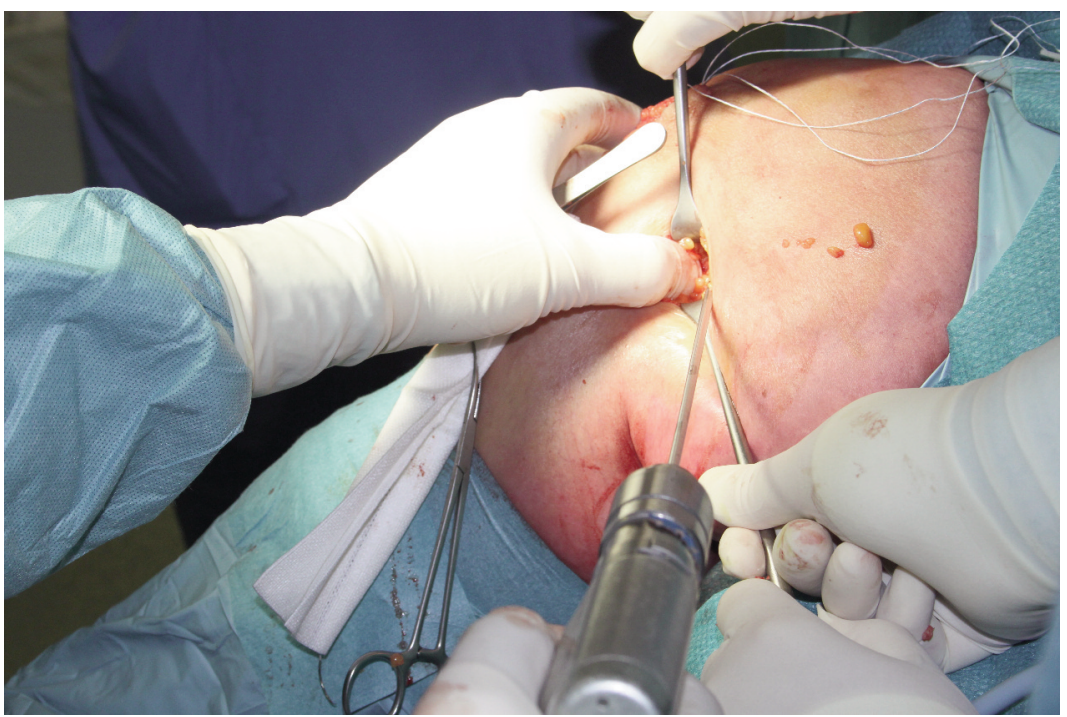

Figure 5. Drilling a tunnel of $1.4 \mathrm{~mm}$ in diameter for the positioning of the two anchors at the femoral edge.

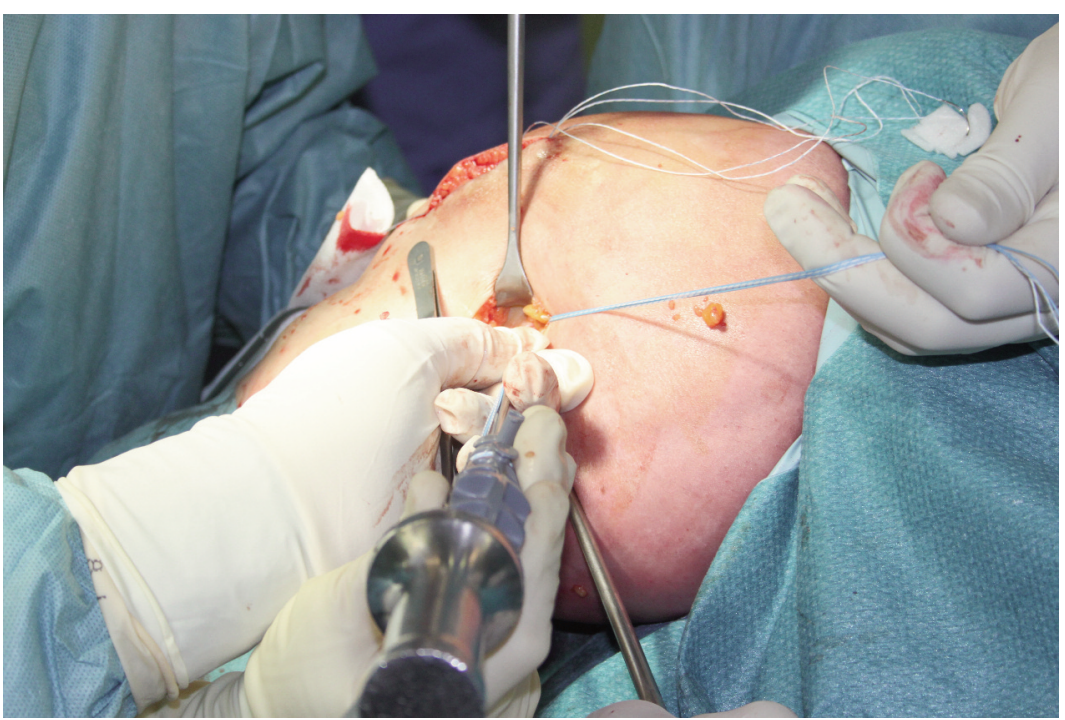

Figure 6. Anchors femoral positioning.

\section{Statistical analysis}

Data are described as number, percentage when quantitative or median, range, media and standard deviation when qualitative. Possible differences between scores and clinical evaluation pre-post surgery are evaluated using Wilcoxon paired data when quantitative or using Fisher test when quantitative $\mathrm{p}<0.05$ is considered significant. Data analysis were carried out by Stata 13 program (www.stata.com).

\section{Results}

Out of the 19 patients 12 were males and 7 females. Average age at surgery was $25.46 \pm 8.28$ years. Average age at first dislocation episode was $19 \pm 6$ years. Median follow up was 22 months (range 6-39).
A median of 4 dislocations (min: 1 max: 20) per patient were found, all caused by physical activity. No familiarity for patellar instability was reported. No patellar dislocation and complications were found at follow up. A clinical evaluation improvement was recorded at follow up: all patients achieved a full range of knee motion; apprehension sign from $89 \%$ positivity (17 cases) decreased up to $11 \%$ ( 2 cases); glide test from 100\% (19 cases) positivity decreased up to $0 \%$ (0 cases).

Median VAS score decreased significantly $(p<0.05)$ from a median pre-operative value of 8 (min: 5 max: 10 ) to 2 (min: 0 max: 7 ). Mean Kujala score improved significantly $(p<0.05)$ from $65.23 \pm 18.64$ pre-operatively to $94.69 \pm 6.40$. Mean Lysholm score improved significantly $(\mathrm{p}<0.05)$ from $64.30 \pm 19.29$ pre-operatively to $94.72 \pm 4.02$. Mean Tegner score decreased from $6.15 \pm 1.06$ pre-operatively to $5.69 \pm 0.85$. 

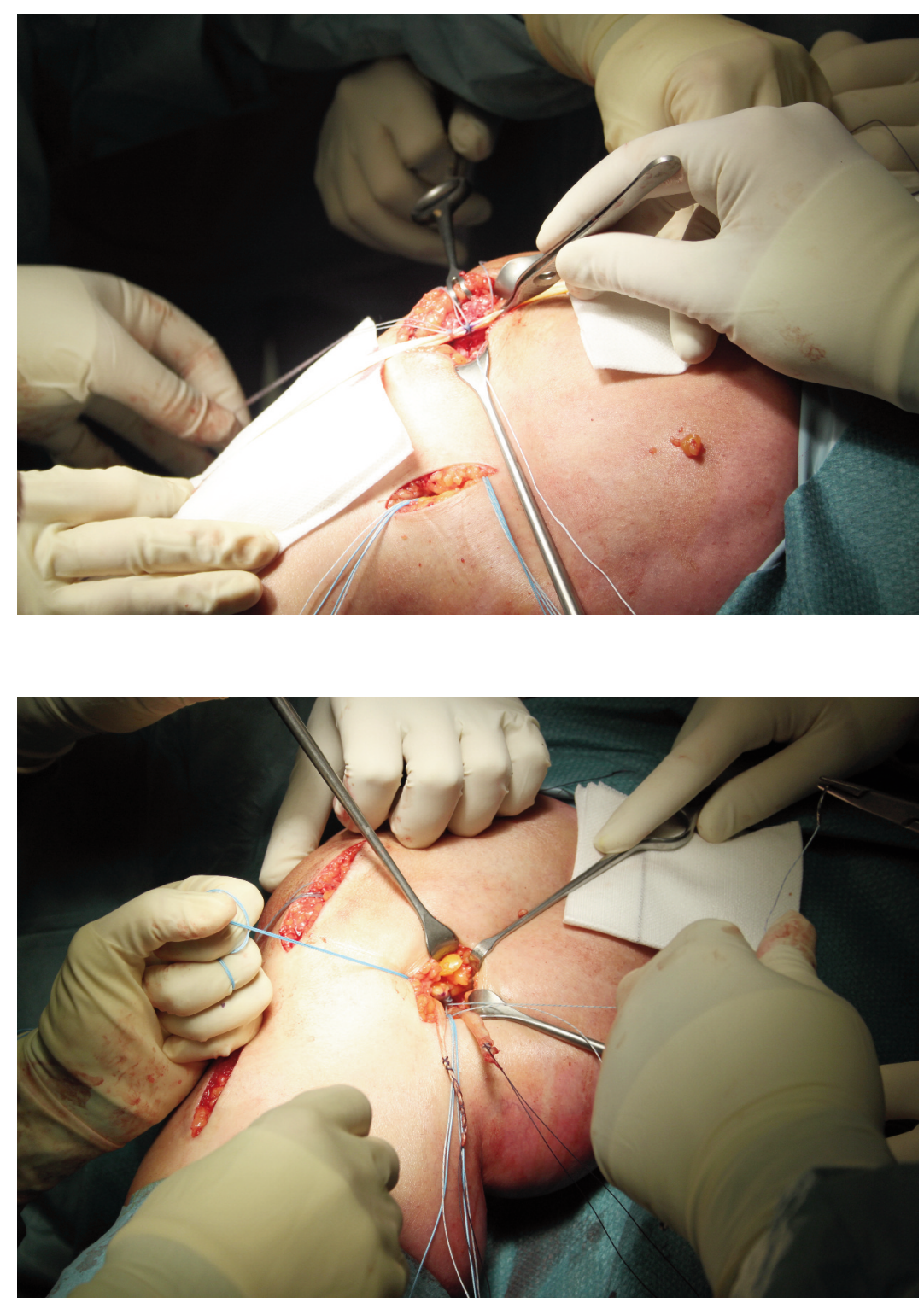

Figure 7. Graft positioning at the medial patellar edge.
Figure 8. Graft positioning at the Schottle's point.

\section{Discussion}

From our preliminary study, the MPFL reconstruction using Juggerknot ${ }^{\mathrm{TM}}$ (BIOMET, Warsaw, Indiana) suture anchors is a valid, safe and effective surgical procedure to treat patellar dislocation. The most important result of our study was the significant increase of the main evaluation scales and the considerable improvement of clinical symptoms in patients examined with a 22 months median follow-up (range 6-39) from the MPFL reconstruction surgery. Of the four scales used all, except TEGNER score, showed a statistically significant improvement. Two studies associated with the creation of bone tunnels and the use of suture anchors ${ }^{17,18}$ allowed us to evaluate the biomechanical characteristics of the MPFL reconstruction technique.
Mountney et al. ${ }^{18}$ have indeed observed that, in relation to the tensile strength of the MPFL, there is no significant difference between the technique with suture anchors and that with tunnel. Even in the study of Hapa et al. ${ }^{17}$ there have been no differences between the two techniques. However, there was a MPFL lower stiffness in the suture anchor group with respect to the bone tunnel group. Russ et al. ${ }^{19}$ showed that interference screw fixation to the medial patella was significantly stronger than suture anchor fixation when comparing the ultimate failure load and stiffness. Nevertheless suture anchor fixation may be sufficient when compared with the native MPFL. Panni et al. ${ }^{20}$, in the 45 patients undergoing reconstruction of the MPFL, had an increase of the main evaluation scales (Kujala from 56.7 pre-operatively to 86.8, Lysholm from 57.6 pre-operatively to 88.1 ) and reported the 
presence of one of the most dangerous complications in reconstruction the MPFL, the fracture of the patella. Christiansen et al. ${ }^{21}$, in 44 subjects investigated with MPFL reconstruction, showed an increase of Kujala score from 46 pre-operatively to 84 , presenting four episodes of dislocation after surgery. These two works have been made on the MPFL reconstruction by creating bone tunnels. The study by Song et al. ${ }^{22}$, carried out on 24 subjects with a suture anchor patellar MPFL reconstruction, showed an increase of Kujala score from 52 pre-operatively to 90.9 and of Lysholm score from 49.2 pre-operatively to 90.9 . It also showed no complication or postoperative dislocation episode. The meta-analysis review of Shah et al. ${ }^{23}$ showed a good success rate of MPFL reconstruction, with a complication rate and surgical failure in only $26.1 \%$ of cases. This percentage falls further $(21.6 \%)$ when considering only the surgical techniques using sutures, while increases slightly $(29.8 \%)$ in those with bone tunnels. In our study, none of the 19 patients analysed showed postoperative complications. Comparing our data with the literature $20-22$, we can say that our technique is similar to other suture anchor surgical techniques and has a lower risk of complications than the techniques associated with bone tunnels. Our surgical technique, using small suture anchors and a milling cutter of small diameter $(1.4 \mathrm{~mm})$, leads to the following advantages: a) prevents tunnelling of the patella, which is the main cause of fracture; $b$ ) ensures a stable mechanical fixation of the graft; c) allows a ligament anatomical, physiological and functional reconstruction; d) the less aggressive surgery to bone structures favours a more rapid rehabilitation. Among all 4 evaluation scales used, the only one that had a slight decrease was the TEGNER score.

However this figure is perfectly aligned with the case studies reported in the literature, where the return to the previous sport physical activity level after one or more episodes of patellar instability is limited to about $2 / 3$ of patients, regardless of treatment 24,25 . This explains why, of our 19 subjects observed, 5 (26\%) were not able to collocate with the same level of preoperative sport efficiency.

The main limitations of our study were the low number of subjects examined, retrospective study with the lack of a control group and the short postoperative follow-up.

\section{Conclusion}

The MPFL is the primary medial stabilizer of the patella ${ }^{5-8}$. MPFL reconstruction has proved proper procedure for OPD patients ${ }^{4}$. In this preliminary study, a clinical assessment of patients undergoing surgery with the Juggerknot ${ }^{\mathrm{TM}}$ (BIOMET, Warsaw, Indiana) suture anchor fixation in MPFL reconstruction, has shown promising results, revealing easy to apply, effective and safe for patients.

Randomized controlled trials are needed to evaluate the efficacy and validity of our long-term technical and compare it with the other techniques in literature.

\section{Conflict of interests}

The Authors declare that they have no conflict of interests regarding the publication of this paper.

\section{References}

1. Fithian DC, Paxton EW, Stone ML, et al. Epidemiology and natural history of acute patellar dislocation. Am J Sports Med. 2004;32(5):1114-1121.

2. Cofield RH, Bryan RS. Acute dislocation of the patella: results of conservative treatment. J Trauma. 1977;17(7):526-531.

3. Zaffagnini S, Grassi A, Muccioli GMM, et al. Medial patellotibial ligament (MPTL) reconstruction for patellar Instability. Knee Surg Sports Traumatol Arthrosc. 2014;22:2491-2498.

4. Smith TO, Walker J, Russel N. Outcomes of medial patellofemoral ligament reconstruction for patellar instability: a systematic review. Knee Surg Sports Traumatol Arthosc. 2007;15 (11):1301-1314.

5. Conlan T, Garth WP Jr, Lemons JE. Evaluation of the media soft-tissue restraints of the extensor mechanism of the knee. $J$ Bone Joint Surg Am. 1993;75(5):682-693.

6. Desio SM, Burks RT, Bachus KN. Soft tissue restraints to lateral patellar translation in the human knee. Am J Sports Med. 1998;26(1):59-65.

7. Fithian $\mathrm{DC}$, Paxton $\mathrm{EW}$, Cohen $\mathrm{AB}$. Indications in the treatment of patellar instability. J Knee Surg. 2004;17(1):47-56.

8. Hautamaa PV, Fithian DC, Kaufman KR, Daniel DM, Pohlmeyer AM. Medial soft tissue restraints in lateral patellar instability and repair. Clin Orthop Relat Res. 1998;(349):174-182.

9. Sherman SL, Plackis AC, Nuelle CW. Patellofemoral anatomy and biomechanics. Clin Sports Med. 2014;33(3):389-401.

10. Burks RT, Desio SM, Bachus KN, Tyson L, Springer K. Biomechanical evaluation of lateral patellar dislocations. Am J Knee Surg. 1998;11(1):24-31.

11. Sallay PI, Poggi J, Speer KP, Garrett WE. Acute dislocation of the patella. A correlative pathoanatomic study. Am J Sports Med. 1996;24(1):52-60.

12. Schottle PB, Fucentese SF, Romero J. Clinical and radiological outcome of medial patellofemoral ligament reconstruction with a semitendinosus autograft for patella instability. Knee Surg Sports Traumatol Arthrosc. 2005;13(7):516-521.

13. Panni AS, Alam M, Cerciello S, Vasso M, Maffulli N.Medial patellofemoral ligament reconstruction with a divergent patellar transverse 2-tunnel technique.Am J Sports Med. 2011;39 (12):2647-2655.

14. Ronga M, Oliva F, Longo UG, Testa V, Capasso G, Maffulli N.Isolated medial patellofemoral ligament reconstruction for recurrent patellar dislocation. Am J Sports Med. 2009;37(9): 1735-1742

15. Carmont MR, Maffulli N.Medial patellofemoral ligament reconstruction: a new technique.BMC Musculoskelet Disord. 2007;28;8:22.

16. Padulo J, Oliva F, Frizziero A, Maffulli N. Muscles, Ligaments and Tendons Journal. Basic principles and recommendations in clinical and field science research. MLTJ. 2013;3(4):250252.

17. Hapa O, Akşahin E, Özden R, et al. Aperture fixation instead of transverse tunnels at the patella for medial patellofemoral ligament reconstruction. Knee Surg Sports Traumatol Arthrosc. 2012;20(2):322-326.

18. Mountney J, Senavongse W, Amis AA, Thomas NP. Tensile strength of the medial patellofemoral ligament before and after repair or reconstruction. J Bone Joint Surg. 2005;87(1) 36-40.

19. Russ SD, Tompkins M, Nuckley D, Macalena J. Biomechani- 
cal comparison of patellar fixation techniques in medial patellofemoral ligament reconstruction. Am J Sports Med. 2015;43(1):195-199.

20. Panni AS, Alam M, Cerciello S, Vasso M, Maffulli N. Medial patellofemoral ligament reconstruction with a divergent patellar transverse 2-tunnel technique. Am J Sports Med. 2011;39 (12):2647-2655.

21. Christiansen SE, Jacobsen BW, Lund B, Lind M. Reconstruction of the medial patellofemoral ligament with gracilis tendon autograft in transverse patellar drill holes. Arthroscopy. 2008; 24(1):82-87.

22. Song SY, Kim IS, Chang HG, Shin JH, Kim HJ, Seo YJ. Anatomic medial patellofemoral ligament reconstruction using patellar suture anchor fixation for recurrent patellar instability. Knee
Surg Sports Traumatol Arthrosc. 2014;22(10):2431-2437.

23. Shah JN, Howard JS, Flanigan DC, Brophy RH, Carey JL, Lattermann C. A systematic review of complications and failures associated with medial patellofemoral ligament reconstruction for recurrent patellar dislocation. Am J Sports Med. 2012;40 (8):1916-1923.

24. Fisher B, Nyland J, Brand E, Curtin B. Medial patellofemoral ligament reconstruction for recurrent patellar dislocation: a systematic review including rehabilitation and return-to-sports efficacy. Arthroscopy. 2010;26(10):1384-1394.

25. Ménétrey J, Putman S, Gard S. Return to sport after patellar dislocation or following surgery for patellofemoral instability. Knee Surg Sports Traumatol Arthrosc. 2014;22(10):23202326. 